\title{
Secondary Electrospray Ionization of Complex Vapor Mixtures. Theoretical and Experimental Approach
}

\author{
Guillermo Vidal-de-Miguel, ${ }^{1,2}$ Ana Herrero ${ }^{1}$ \\ ${ }^{1}$ SEADM S.L., Valladolid, Spain \\ ${ }^{2}$ Energy and Fluid Mechanics Engineering Department, Valladolid University, Valladolid, Spain
}

\begin{abstract}
In secondary electrospray ionization (SESI) systems, gaseous analytes exposed to an electrospray plume become ionized after charge is transferred from the charging electrosprayed particles (the charging agent) to the vapor species. Currently available SESI models are valid for simplified systems having only one type of electrosprayed species, which ionizes only one single vapor species, and for the limit of low vapor concentration. More realistic models require considering other effects. Here we develop a theoretical model that accounts for the effects of high vapor concentration, saturation effects, interferences between different vapor species, and electrosprays producing different types of species from the liquid phase. In spite of the relatively high complexity of the problem, we find simple relations between the different ionic species concentrations that hold independently of the particular ion source configuration. Our model suggests that an ideal SESI system should use highly concentrated charging agents composed preferably of only one dominating species with low mobility. Experimental measurements with a $\mathrm{MeOH}-\mathrm{H}_{2} \mathrm{O}-\mathrm{NH}_{3}$ electrospray and a mixture of fatty acids and lactic acid served to test the theory, which gives good qualitative results. These results also suggest that the SESI ionization mechanism is primarily based on ions rather than on charged droplets.
\end{abstract}

Key words: Electrospray, Vapor ionization, Vapor analysis, SESI

\section{Introduction}

$\mathrm{T}^{\mathrm{s}}$ he ionization of vapors by means of electrosprays or coronas is playing an increasingly important role in vapor analysis applications such as explosives detection or breath analysis.

Secondary electrospray ionization (SESI), first introduced by Fenn et al. [1-3] and later worked on by $\mathrm{Wu}$ et al. [4], takes place when an electrospray cloud (the charging agent) comes into contact with a gas carrying vapors (the sample vapors), resulting in the formation of vapor ions (sample ions) at atmospheric pressure. Unlike corona discharges or radioactive ionizers, SESI is a soft ionization process. Furthermore, the charging agent's affinity can be selected

Correspondence to: Guillermo Vidal-de-Miguel; e-mail: guillermo.vidal@ seadm.com so as to ionize only vapors below certain ionization affinity. This limits the types of molecules that can be ionized (only polar molecules), but has the advantage of reducing chemical noise produced by the carrying gas or other undesired vapors. Recent experiments $[5,6]$ show sensitivities below the ppt level, and ionization probabilities $p_{i}$ of the order of $10^{-4}$, as defined in equation (1):

$$
p_{i}=n_{s} / N_{s}
$$

where $n_{s}$ is the concentration of sample ions and $N_{s}$ is the concentration of sample neutral vapors.

Whether vapor charging takes place directly via contact with the droplets, or indirectly by charge transfer from ions released from the droplets, remains unclear [7]. Recent studies [8] using a secondary electrospray ionization, differential mobility analyzing and mass spectrometry (SESI-DMA-MS) analyzer with a charging electrospray 
of formic acid, and vapors containing different types of amides, suggest that the ions option is more likely to be happening. In this case, the charge transmission reaction taking place would be: formiate ${ }^{-}+$amide $\rightarrow$ amide $^{-}+$ formic. The exact charge transfer mechanism in a more general case still depends on many factors. However, for small ES droplets or at high temperature, when the droplets are quickly evaporated, it is reasonable to hypothesize that vapor charging will be attributable primarily to charging ions rather than droplets.

The ionization probability measured by Mesonero et al. [6], though achieving limits of detection in the range of parts per trillion, is as low as $p_{i}=10^{-4}$. As explained by de la Mora [7], the major factor limiting the ionization probability when the sample is ionized by means of a concentrated unipolar charge emitter such as electrospray is the space charge effect, which dilutes the ions at the same rate as sample ions are produced. De la Mora noticed that the strong ionic velocities produced by the space charge needed to be accounted for [7]. According to de la Mora's reasoning, sample ions are produced at a rate proportional to the concentration of charging ions $\left(n_{c}\right)$ but, because space charge is also produced primarily by the charging ions, sample ions are diluted at a rate also proportional to the concentration of charging ions. In stationary conditions, these two effects reach an equilibrium in which the balance between the production of new sample ions and the dilution attributable to space charge leads to a simple expression for the ionization probability:

$$
p_{i}=\frac{k_{c s} \varepsilon_{0}}{Z_{s} \cdot e}
$$

[7]where $e$ is the charge of the ion, $\varepsilon_{0}$ is the permittivity of the vacuum, $Z_{s}$ is the mobility of the sample ion, and $k_{c s}$ is the reaction rate coefficient for the charge transfer reaction from the charging ions to the sample molecule. It is remarkable how such a simple expression might describe a phenomenon apparently so complicated. Further comparisons of the measured values of $p_{i}$ with those predicted by equation 2 show that this equation agrees, at least in the order of magnitude, with experimental data $[6,7]$.

The low theoretically predicted value of $p_{i}$ indicates that the ionization probability achievable in practice is highly limited. On the other hand, the fact that the amount of sample ions is proportional to the amount of available molecules can be useful to estimate quantitatively the concentration of sample vapors. Unfortunately, experiments using complex mixtures of vapors show that there is a dependence of the sample ions produced on the concentration of other vapors. For instance, following the experiments by Martínez-Lozano $[9,10]$, we tried to improve background levels and enhance the sensitivity of an online skin odor analyzer by collecting the skin vapors in an enclosed chamber through which clean nitrogen was first put in contact with the skin and was then directed to the SESI ionizer. When we brought the skin closer to the apparatus, instead of improving the sensitivity, the signals of many species originally present in the background dropped, whereas lactic acid produced a huge signal. We concluded that lactic acid, which was more concentrated in our closed set-up, could be interfering with the ionization of other compounds.

While de la Mora's model [7] is valid in the greatly diluted limit, the purpose of the present study is to develop a theory with which to estimate the ionization probability in more complex samples. Here, we consider finite vapor concentration and associated suppression and saturation effects. In multicomponent vapors, the more concentrated species tend to consume the available charging agents as well as minority ionized vapors having lower ion affinity. These effects lower the probability of ionization, preclude quantification of original vapor concentration, and could even blind the analyzers which, as a result, cannot detect the less concentrated species, which is often of more interest for analysis. These effects are studied here, both theoretically and experimentally. In particular, we deal with the situations where a major species interferes with and reduces the ionization probability of the minor sample species, and where the ionization source is working near saturation. Despite the complexity of the subject, we find that in most situations of interest, the concentration of the different ionized vapors can be expressed as a function of the concentration of charging ions independently of the particular ionizer configuration. As an extension of the theory, we are also analyzing the effect of electrospray plumes emitting complex mixtures of charging ions and other types of useless particles. Our model suggests that an ideal SESI should use highly concentrated charging agents composed preferably of only one dominating species having low mobility. The results also suggest that in $\mathrm{MeOH}-\mathrm{H}_{2} \mathrm{O}-\mathrm{NH}_{3}$ electrosprays, ions rather than charged droplets are primarily responsible for the ionization of lactic acid vapors. Together with this theoretical approach, experimental measurements are also carried out to quantify the effect of lactic acid in a group of fatty acids already found in the skin vapors, and to check the validity of the theory.

\section{Methods}

The objective of the experiments is to provide qualitative data for the behavior of the specific studied species as well as some experimental support for the proposed theory. Vapors generators allow the production of vapors of the desired species at minute, controlled, and known concentrations. In a vapor generator, an electrospray of ultra pure methanol and water (1:1), and a known concentration of a given species (the sample), is produced in a heated chamber where the nanodrops are mixed with a flow of gas (nitrogen in this study). Nanodrops are quickly evaporated and the sample species stay vaporized and neutral within the carrying gas. The flow of gas is controlled and measured using a flow-meter. Assuming a Poiseuille flow, the pressure drop along the electrospray capillary permits us to control the flow of the desired species. The electrospray thus used to 
generate vapors at minute concentrations is called secondary electrospray $[5,6]$. Two vapor generators are coupled with an ionization chamber, which is in turn coupled to an inhouse modified Qstar XL used in single TOF MS mode. In this case, the species used have been lactic acid and several fatty acids. In order to prevent sample vapor deposition in the inner walls of the tubes, every part of the system is heated using proportional integral derivative (PID) controllers. The ionization chamber is composed of a plate holding an electrospray needle and an inlet and an outlet tube placed upstream of the curtain plate of the MS atmospheric pressure interface (API). This scheme is similar to that already proposed by Martínez-Lozano [11] except for a tube inside the chamber that guides the sample flow towards the ESI plume, allowing lower flow-rate operation (2 lpm versus $6 \mathrm{lpm}$ in [11]). The primary electrospray at the ionization chamber produces negative ions (in negative mode) resulting from an ammonium-methanol-water dissolution. Sample vapors flow continuously through the ionization chamber; vapors enter through the inlet tube, pass in front of the electrospray tip, and exit, along with the counterflow gas, through the outlet tube, so that the cloud of charging agents produced by the charging electrospray is in close contact with the sample gas before ions are driven towards the analyzer inlet by the strong electric fields produced by the electrospray. Figure 1 shows schematically the hardware used for the experiments in this study.

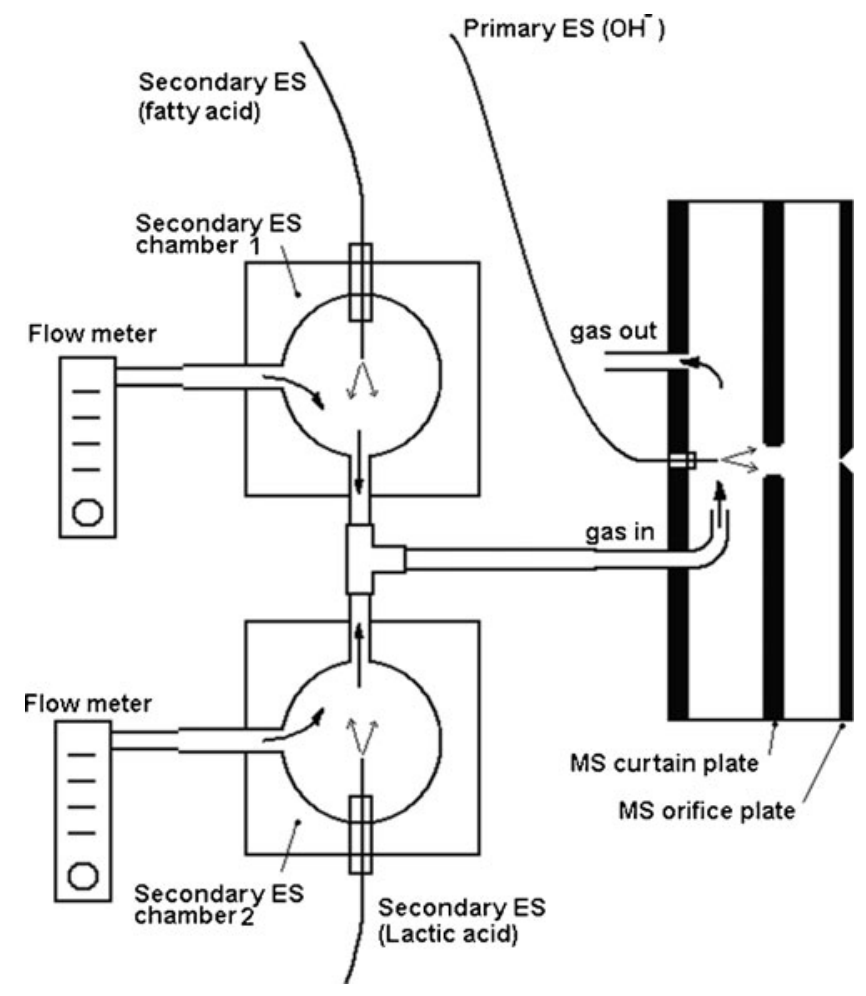

Figure 1. Hardware used to study the behavior of an electrospray-based vapor ionization system comprising two vapor generators
The purpose of these experiments is to establish the relationship between the concentration of vapors and the concentration of ions produced in the ionization region. Vapor concentration can be easily controlled. In this respect, two main regions can be distinguished: (1) a sample gas region affected by the vapor jet, and (2) a clean gas region, affected by the counterflow gas. Turbulence in the tubes ensures that the concentration of vapors in the sample gas region is approximately uniform. Variations of the vapor concentration can be expected only in the narrow shear layer between the sample gas jet and the counterflow gas region. Sample ions are only produced in the sample gas region where charging agents are in contact with the sample vapors. Our goal in this study is to find relationships between vapor and ion concentrations in this region. But how can we infer the concentration of sample ions here if we are detecting them after they have crossed the clean gas region, the vacuum interface, and all the mass spectrometer optics?

The rate of ions ingested by the MS is proportional to their concentration at the MS inlet. Once ions reach the sonic MS inlet, they pass through the API interface, the quadrupoles, and the TOF system until they are finally detected. Most of them are lost in this journey, but we hypothesize that for the range in which we are interested, the fraction reaching the sensor is fixed (independent of $\mathrm{m} / \mathrm{z}$ ). In this respect, we know that the QStar XL mass analyzer gain is approximately constant through this mass range [8]. Because the gas is clean in the clean region (from the sample gas region to the MS inlet), ions are only affected by space charge repulsion, but this effect can be minimized as long as the electrostatic field is stronger than the Coulomb repulsion. And this can be easily achieved as long as the primary electrospray tip is separated from the curtain plate to ensure that charging ions are already sufficiently diluted when they reach the clean region. We have, therefore, positioned the electrospray tip in the farthest position possible $(2 \mathrm{~cm}$ from the curtain orifice). Under these conditions, the concentration of sample ions reaching the sonic MS inlet is approximately equal to the concentration in the boundary separating the sample and counterflow regions, and therefore the ion signal is proportional to the concentration of ions in this boundary.

\section{Theory}

\section{Charging Vapors in the Linear Regime, Theoretical Approach}

Under the assumptions already explained by de la Mora (stationary regime, negligible fluid velocity, uniform vapor concentration, negligible loss of available vapor molecules and charging ions attributable to sample ion production chemical reactions, and negligible concentration of sample ions on the electric fields), we already know that sample ion concentration reaches the equilibrium given by equation 2 . But is this equilibrium solution valid for all configurations? 
If it were so, the ionization efficiency of all charge transfer techniques should be the same no matter the configuration of the ionizer, as long as the same charging ions are used. Applying the mass conservation equation for each species (the charging and the sample ions), the time derivative of the concentration of each ion is equal to the production rate of ions minus the convective dilution due to space charge. Assuming that the proportion of charging ions lost by charge transfer reactions is negligible compared with space charge dilution, the set of equations for the two species reads as:

$$
\begin{aligned}
& \frac{d n_{s}}{d \tau_{s}}=k_{c s} N_{s} n_{c}-n_{s} \nabla \cdot\left(Z_{s} \vec{E}+\vec{V}_{f}\right) \\
& \frac{d n_{c}}{d \tau_{c}}=-n_{c} \nabla \cdot\left(Z_{c} \vec{E}+\vec{V}_{f}\right)
\end{aligned}
$$

where $\boldsymbol{E}$ is the electric field, $\boldsymbol{V}_{f}$ is the fluid velocity of the gas dragging the ions, and $\tau$ is the time of each infinitesimally small volume following the ionic streamlines at a velocity $\boldsymbol{V}_{\boldsymbol{f}}+Z \boldsymbol{E}$. Note here that $d n / d \tau$ in equations 3, not to be confused with Eulerian notation, refers to the Lagrangian time derivative of the concentration of an infinitesimally small volume following the ions at the same speed of the ions. Although $d n_{s} / d \tau$ differs from zero, the configuration might be in a stationary state. Because each type of ion has a different mobility, the streamlines of species is different and $\tau$ cannot be directly simplified. But if the fluid velocity is negligible, or if both the sample ion and the charging ion have the same mobility, they both follow the same trajectory (though they might travel at different speeds). Fortunately, these conditions apply in our ionizer, at least in the sample gas region, where the flow travels smoothly, and very intense electric fields are applied producing ionic velocities as high as $100 \mathrm{~m} / \mathrm{s}$ (near the electrospray tip). Under these conditions, the set of equations can be simplified and further integrated (details in Appendix I), and the relation between the concentration of sample and charging ions along a generic streamline can be expressed as:

$$
n_{s}=n_{s}^{*}\left(1-\frac{n_{c}}{n_{c 0}}\right)+n_{s 0} \frac{n_{c}}{n_{c 0}}
$$

where $n_{s}^{*}=N_{s} \frac{k_{c s} \varepsilon_{0}}{Z_{s} \cdot e}$ and $n_{s 0}$ and $n_{c 0}$ are, respectively, the concentrations of sample and charging ions in a reference section of the streamline.

In the case of an electrospray, two main regions have to be distinguished. In the first region, the apex of the electrospray emits nearly monodisperse droplets, which quickly evaporate and reduce their size, producing a sequence of Coulombic explosions [12]. This process is eminently dynamic and the shape of the charging agents varies, but eventually the charging agents reach a stable condition of well defined clusters. Equation 4 is only valid in the second region, where the composition of the charging agent is stable. But, in the case of interest, droplet evaporation is a very fast process taking place in a very narrow region near the cone apex. As the first region is very small, the charging agents entering the second region are highly concentrated and tend to be quickly diluted by Coulombic repulsion. As a result, it is reasonable to assume that the inequality $n_{c} / n_{c 0}<<1$ holds in the second region. Obviously, there are no clear boundaries between the two regions defined, but the assumption $n_{c} / n_{c 0}<<1$ permits us to neglect the effect of the first region. Under these circumstances, equation 4 reduces to $n_{s}=n_{s}{ }^{*}$, yielding the ionization probability previously predicted [7].

In the case of a corona discharge, two main regions would also have to be distinguished, one region closer to the needle tip where a bipolar plasma is formed, and the other farther from the needle tip, where only ions of one polarity are expelled. The same reasoning referred to above would apply to the case of coronas as long as the plasma region is small.

The effect of diffusion can be ignored because, for the case of interest, the convective velocity is much higher than the diffusive velocity. The convective velocities are of the order $Z E$, while diffusive velocity $\left(V_{D}\right)$ is, using the Einstein's relation, $V_{D}=Z k_{B} T / e \cdot l$, where $k_{B}$ is the Boltzmann constant, $T$ is the temperature of the gas, $Z$ is the mobility, $E$ is the electric field, and $l$ is the size of the region studied. The ratio of the two velocities is the dimensionless parameter $P=E \cdot l /\left(k_{B} T / e\right)$ which, in the case of an electrospray, is typically very high $(E \cdot l$ is around a few $\mathrm{kV}$ while $k_{B} T / e \sim 0.026 \mathrm{~V}$ at ambient temperature).

\section{Charging Vapors Comprising a Major Species and a Minor Species, Theoretical Approach}

One interesting consequence of expressions 2 and 4 is that the concentration of sample ions is, on a first approximation, independent of the concentration of charging ions as long as $n_{c} / n_{c 0}<<1$. The charging ions produce a strong background in the analyzer, which it would be better to minimize. Naturally one concludes that the charging ions should be diluted as much as possible in order to achieve this, and this can be achieved by simply positioning the emitter farther away from the analyzer inlet. But charging ions cannot be diluted too much because, eventually, the ions would be depleted and no new sample ions would be produced. So, a new question arises: How much can the charging ions be diluted to improve the background before losing performance? Answering this question requires considering real world samples often composed of substances at very different concentrations. A common scenario is a sample containing a major substance and other minor substances more difficult to detect. For instance, complex biomedical samples such as skin emanations include fatty acids at much smaller concentrations than lactic acid [9]. In this example, as one would expect, the minimum concentration of charging ions would be limited by the lactic rather than the fatty acids.

One objective of this study is to describe and predict the behavior of a sample comprising one major species and 
other minor species. This could be useful to establish how much one can dilute charging ions before performance loss. It could also be useful for estimating the interference of different species and for calculating the original sample concentration from measurements where the ionized species interfere with each other.

As minor species at low concentrations do not affect other species, only one minor species will be considered in the theoretical approach. In order to clarify the nomenclature, and because the major species reduces the signal produced by the minor species, the major species is termed buffer (subscript $b$ ), and the minor species is simply termed sample (subscript ${ }_{s}$ ). Buffer species might interfere with the ionization of the sample in two main ways. Buffer vapors can deplete the amount of charging ions available by charging to buffer charge transfer reactions, and they can also neutralize sample vapors by sample to buffer charge transfer reactions. For simplicity, only one charging species and one buffer species will be considered here.

In the regime of interest, the electric field is produced by both the charging and the buffer ions, and the production of buffer ions is also reflected in a nonnegligible destruction of charging ions. The amount of sample vapors will be considered sufficiently small so that its effect on both the neutralization of charging ions and the electric field are negligible. Sample ions are produced by transfer reactions from charging ion to the sample vapor and are neutralized by charge reactions from sample ions to neutral buffer vapors. The set of equations governing the behavior of the charging, the buffer, and the sample ions becomes:

$$
\begin{aligned}
& \frac{d n_{s}}{d \tau_{s}}=k_{c s} N_{s} n_{c}-k_{s b} n_{s} N_{b}-n_{s} \nabla \cdot\left(Z_{s} \vec{E}+\vec{V}_{f}\right) \\
& \frac{d n_{c}}{d \tau_{c}}=-k_{c b} N_{b} n_{c}-n_{c} \nabla \cdot\left(Z_{c} \vec{E}+\vec{V}_{f}\right) \\
& \frac{d n_{b}}{d \tau_{b}}=k_{c b} N_{b} n_{c}-n_{b} \nabla \cdot\left(Z_{b} \vec{E}+\vec{V}_{f}\right) \\
& \nabla \vec{E}=\frac{e}{\varepsilon_{0}}\left(n_{c}+n_{b}\right)
\end{aligned}
$$

where $n_{b}$ is the concentration of buffer ions, $N_{b}$ is the concentration of buffer vapors, $Z_{b}$ is the mobility of buffer ions, and $k_{c b}$ and $k_{s b}$ are the reaction rate constants for charge transfer from the charging ions to the buffer molecule, and from the sample ions to the buffer molecules, respectively.

Again, if the fluid velocity is negligible, or if the sample, the buffer, and the charging ions have the same mobility, all ions follow the same streamlines (though they might have different velocities). The variable $\tau$ can then be further simplified and the concentration of both buffer and sample ions can be expressed as a function of the charging ion (see details in Appendix II):

$$
\begin{aligned}
\frac{d \eta_{b}}{d \eta_{c}} & =\frac{\eta_{c}-\eta_{b}\left(\eta_{c}+\eta_{b}\right)}{-\eta_{c} Z_{b c}-\eta_{c}\left(\eta_{c}+\eta_{b}\right)} \\
\frac{d \eta_{s}}{d \eta_{c}} & =\frac{\eta_{c}-\eta_{s} Z_{b c} \kappa-\eta_{s}\left(\eta_{c}+\eta_{b}\right)}{-\eta_{c} Z_{b c}-\eta_{c}\left(\eta_{c}+\eta_{b}\right)}
\end{aligned}
$$

where $n_{s}^{*}=N_{s} \frac{k_{c s} \varepsilon_{0}}{Z_{s} \cdot e}$ and $n_{b}^{*}=N_{b} \frac{k_{c b} \varepsilon_{0}}{Z_{b} \cdot e}$ are parameter groups having dimensions of concentration, $\eta_{s}=\eta_{s} / \eta_{s}^{*}, \eta_{c}=$ $\eta_{c} / \eta_{b}^{*}, \eta_{b}=\eta_{b} / n_{b}^{*}$. are dimensionless concentrations, $z_{b c}=Z_{b} / Z_{c}$ is the buffer to charging mobility ratio, and $\kappa=k_{s b} / k_{c b}$. is the ratio of reaction rate constants. Buffer vapor reduces the ionization of minor species by (1) competing for the available charging ions and by (2) neutralizing already ionized minor sample ions. The ratio $\kappa$ represents the relative importance of these two different processes.

This set of equations 7 can be numerically integrated given a set of initial conditions $\left(n_{s 0}, n_{b 0}\right.$, and $\left.n_{c 0}\right)$. In the case of an electrospray having a singular source of charging ions, one would expect to see the asymptotic behavior already predicted by de la Mora [7] near the cone apex, where the concentration of charging ions tends to infinity. To recover this behavior, equations 7 are integrated numerically by starting the integration at the source $\eta_{c} \rightarrow \infty$ with the equilibrium initial condition that $\eta_{s} \rightarrow 1$ and $\eta_{b} \rightarrow 1$.

\section{Charging Vapors when the Electrospray Plume Produces Charging Agents, and Also Charged Particles, which Reduce Performance, Theoretical Approach}

Our discussion has so far been limited to the case where the charging agent is composed of only one ion type. In more realistic situations, the electrospray might produce several types of ions and clusters. Electrosprays tend to produce, once droplets are evaporated, single ions as well as dimers, trimers, and many types of clusters depending on each particular solution [12]. Some of these charging agents will produce ions when combined with the sample vapors, but other species produced by the spray will not react to produce vapor ions, or will simply react differently, producing other adducts that will appear in a different peak in the spectrum. Such species would not produce sample ions, but would still dilute the sample by space charge. How exactly would these species affect our sample? For simplification, only one type of charging ion is considered to produce the desired sample ions, whereas other electrosprayed species would not produce the desired sample ions. The only effect of those useless charged particles is to increase the electrostatic repulsion of the ions.

The objective of the present study is to evaluate the effect of complex electrospray plumes on the probability of ionization. For simplicity, we only consider one sample species at very low concentration (sufficiently low to ignore 
its effects on space charge and charging ion concentration). The set of equations governing the behavior of the charging ions, the useless electrosprayed ions, and the sample ions are:

$$
\begin{aligned}
& \frac{d n_{s}}{d \tau_{s}}=k_{c s} N_{s} n_{c}-n_{s} \nabla \cdot\left(Z_{s} \cdot \vec{E}+\vec{V}_{f}\right) \\
& \frac{d n_{c}}{d \tau_{c}}=-n_{c} \nabla \cdot\left(Z_{c} \cdot \vec{E}+\vec{V}_{f}\right) \\
& \frac{d n_{i}}{d \tau_{i}}=-n_{i} \nabla \cdot\left(Z_{i} \cdot \vec{E}+\vec{V}_{f}\right) \\
& \nabla \vec{E}=\frac{e}{\varepsilon_{0}}\left(n_{c}+\sum n_{i}\right)
\end{aligned}
$$

where the useless electrosprayed ions are denoted with subscript ${ }_{i}, n_{i}$ is the concentration of the useless electrosprayed particle of type $i$, and $Z_{i}$ is its mobility. Again, if all particles travel along the same streamlines, the equations can be simplified (see Appendix III):

$\frac{d n_{s}}{d n_{c}}=\frac{n_{s}^{*} n_{c}-n_{s}\left(n_{c}+\sum n_{i}\right)}{-n_{c}\left(n_{c}+\sum n_{i}\right)}$

$\frac{d n_{i}}{d n_{c}}=\frac{n_{i}}{n_{c}}$

Equation $10 \mathrm{~b}$ can be directly integrated showing that the concentration ratio of each species remains constant: $n_{i} / n_{c}=$ $C_{i}$. Equation 10a can also be integrated analytically yielding:

$$
n_{s}=\frac{n_{s}^{*}}{1+\sum C_{i}}\left(1-\frac{n_{c}}{n_{c 0}}\right)+n_{s 0} \frac{n_{c}}{n_{c 0}}
$$

Equation 11 shows that the ideal single-charging concentration $n_{s}{ }^{*}$ is actually reduced by a potentially large factor $\gamma=1+\sum C_{i}$. This factor shows that charging agents should be composed preferably of only one ion type. Whether this factor could also explain the strong dependence of the ionization probability on other parameters affecting the structure of the charging electrospray plume (such as humidity, temperature, or other parameters) is a question that needs further study.

\section{Results and Discussion}

Both experimental results and theoretical results have to be presented in a way that allows comparison and validation. The theoretical approach shows the relationships between the charging ions and the vapor sample ions. Basically, the numerical solution to equation 7 provides the functions $\eta_{s} \leftrightarrow \eta_{c}$ and $\eta_{b} \leftrightarrow \eta_{c}$ for different values of the mobility ratio $z_{b c}$ and the kinetic ratio $\kappa$. But experimental data provide only vapor concentration and sample ion signals.

In the experiments we have varied the concentration of interfering lactic acid. For a given ionization configuration, the concentration of minor sample ions in the boundary separating the sample gas region and the clean counterflow regions depends on the concentration of the major vapor. On a first approximation, if the charging and buffer ions have similar mobilities, the structure of the electrospray plume and the concentration of total charge, governed by the total emitted current and the mobility of the ions within the plume [13], would be unaffected even if the charge is transferred from one species to another, and the sum of the concentration of charging plus buffer ions would not depend on the concentration of buffer ions. It is therefore reasonable to assume that for $z_{b c}$ close to one, the concentration of charging plus buffer ions in this boundary remains constant ( $n_{\text {tot }}=n_{b}+n_{c}=$ constant, where $n_{\text {tot }}$ is the total amount of available ions). Figure $2 \mathrm{a}$ and $\mathrm{b}$, reconstructed from data obtained by numerical integration of equation 7 , illustrate the fraction of buffer ions among the total amount of available ions $n_{b} / n_{t o t}$ and the dimensionless concentration of sample ions $n_{s} / n_{s}^{*}$ as a function of the dimensionless concentration of buffer vapors $n_{b}^{*} / n_{t o t}$ (note here that $n^{*}{ }_{b}$ is, by definition, proportional to the concentration of buffer vapors). As expected, sample ions produce lower signals, whereas buffer ions produce higher signals and tend toward saturation at increasing buffer vapor concentrations.

Figure $2 b$ shows the results for different values of $\kappa$, and for $z_{b c}=1$ (buffer and charging ions having the same mobility). Curves corresponding to the evolution of the buffer ion are collapsed because its behavior does not depend on $\kappa$. As expected, at higher $\kappa$ (when the buffer neutralizes the minor sample species), the concentration of sample ions falls very quickly, even before charging ions are depleted, and the buffer ions signal still behaves linearly with buffer concentration.

\section{Comparison of Theoretical and Experimental Data}

The data obtained in the experiments is the signal of the sample ions $S_{s}$ (fatty acids) and the buffer ions $S_{b}$ (lactic acid) versus buffer vapor concentration $N_{b}$ at a given sample vapor concentration $N_{s}$. As expected, the behavior of the buffer ion signal at increasing buffer vapor concentrations shows a linear regime, a saturation regime, and a transition regime.

In the saturation regime, the buffer signal is constant $\left(S_{b, \max }\right)$. In this regime, it is reasonable to assume that the signal is proportional to the total concentration of ions available: $S_{b, \max }=n_{t o t} \cdot G$, where $G$ is the gain of the analyzer and is unknown. Assuming that $S_{b}=N_{b} \cdot G$, the dimensionless parameter $n_{b} / n_{\text {tot }}$ can be estimated experimentally as:

$$
n_{b} / n_{t o t}=S_{b} / S_{b, \max }
$$

On the other hand, in the linear regime, the signal is proportional to the concentration of buffer vapors being 
(a)

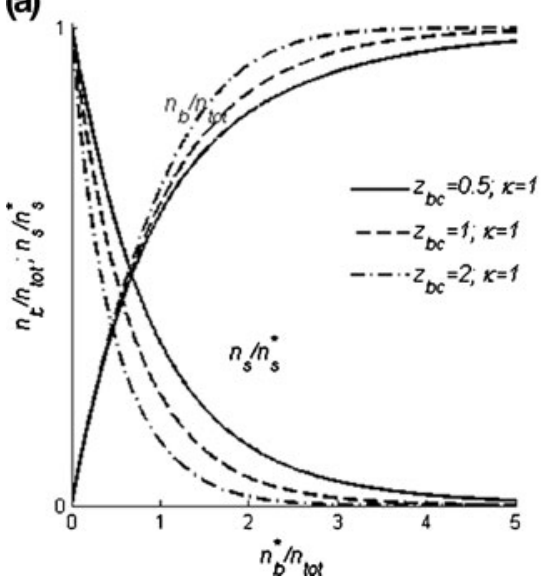

(b)

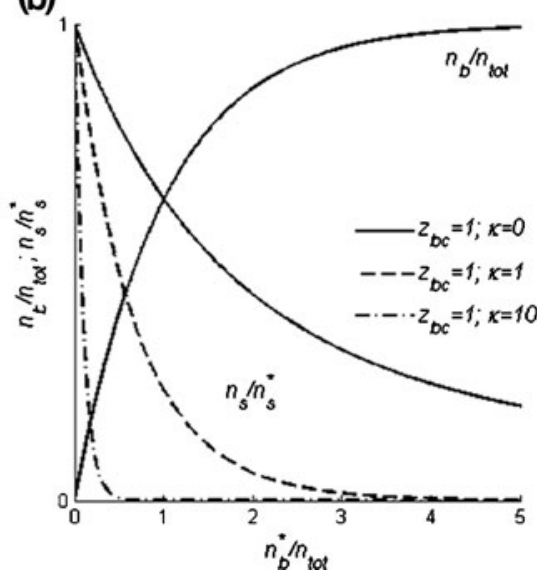

Figure 2. (a) and (b): theoretically calculated evolution of the buffer and the minor sample ion concentrations as a function of the buffer vapor concentration: and dimensionless curves for different values of $z_{b c}$ near $z_{b c}=1$ (a), and for different values of $\kappa(b)$

$S_{b}, l=p_{b, l} G \cdot N_{b}$, where $p_{b, l}$ is the probability of ionization of the buffer species in the linear regime. Though $G$ is unknown, the value $p_{b, l} G$ can be easily calculated by a simple linear regression in the linear region of the $S_{b}\left(N_{b}\right)$ curve. Using the relation between $p_{b, l}$ and $n_{b}^{*}\left(n_{b}=p_{b, l} N_{b}\right.$ by definition), the dimensionless parameter $n_{b}^{*} / n_{\text {tot }}$ can also be estimated experimentally as:

$$
n_{b}^{*} / n_{t o t}=p_{b, l} \cdot G / S_{b, \max } \cdot N_{b}
$$

With regard to the sample ions, the ratio $n_{s} / n_{s}^{*}$ can also be estimated experimentally assuming that in the absence of lactic acid, the concentration of sample ions approaches the ideal concentration $n_{s}^{*}$ and that signals are proportional to ion concentration. The dimensionless parameter $n_{s} / n^{*}{ }_{s}$ can be experimentally estimated as:

$$
n_{s} / n_{s}^{*}=S_{s} / S_{s(N b=0)}
$$

As for the behavior of the buffer ions, our ionizer starts to become saturated at lactic acid concentrations near 10 $\sim 20 \mathrm{ppb}$. Figure 3 shows both the theoretical and the experimental $n_{b} / n_{\text {tot }} \leftrightarrow n_{b}^{*} / n_{\text {tot }}$ curves together. Due to the way experimental data are processed, the behavior for $n_{b}^{*} / n_{\text {tot }} \rightarrow \infty$ and $n_{b}^{*} / n_{\text {tot }} \rightarrow 0$ is constrained and fitted to the theoretical values. Differences between experimental and theoretical behavior can be seen only in the transition region, which should be somewhere near $n_{b}^{*} / n_{\text {tot }} 1$. Note here that the shape of the curve depends only on the parameter $z_{b c}$. In

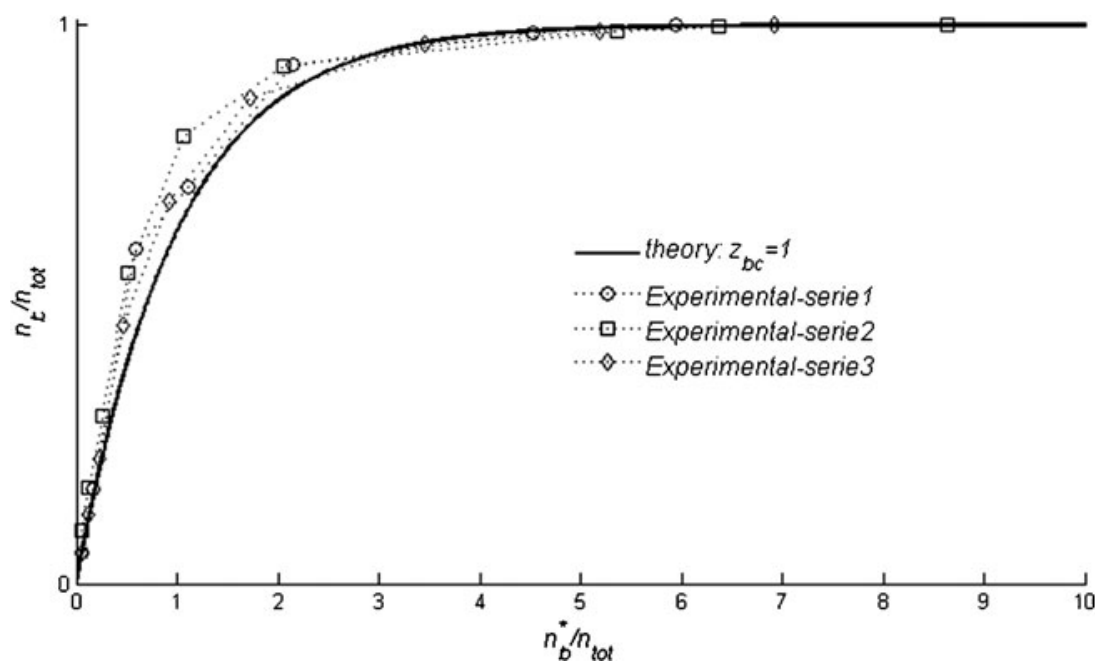

Figure 3. Experimental and theoretical curves. Dimensionless buffer ion concentration as a function of dimensionless buffer vapor concentration 


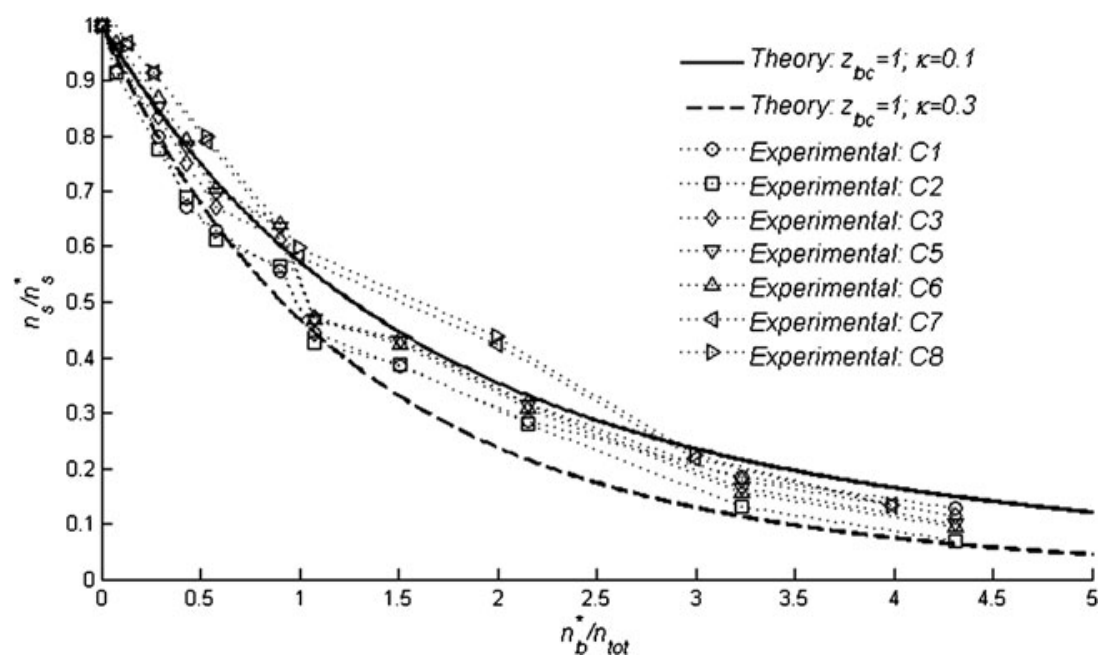

Figure 4. Experimental and theoretical curves. Dimensionless sample ion concentration as a function of dimensionless buffer vapor concentration

this case, the value for $z_{b c}=1$ has been chosen arbitrarily under the hypothesis that lactic acid ions and charging ions would have a fairly similar mobility. It would have been useful to know the exact charging agent but, unfortunately, we could not find a dominating peak in the MS spectrum inversely correlated with the lactic acid signal, and we still do not know the exact ionization mechanism. We hypothesize that the ionization agent is very light and its mass is too small to be detected by our mass spectrometer (almost blinded for ions smaller than $30 \sim 40 \mathrm{Da})$. However, although the charging agent still remains unknown, the behavior shown in Figure 3 suggests that the mobility of the charging agent is similar to or slightly smaller than that of the lactic acid, so the charging agent might be a cluster comprising an $\mathrm{OH}^{-}$ion and a few molecules of water or methanol. This result is in agreement with recent studies by Martínez-Lozano [8], and also supports the original hypothesis that we cannot detect the charging agent simply because it is too small for our analyzer.

Figure 4 shows the curves $n_{s} / n_{s}^{*} \leftrightarrow n_{b}^{*} / n_{\text {tot }}$ (sample ion concentration as a function of buffer vapor concentration) evaluated both theoretically and experimentally for different saturated fatty acids at concentrations near 1 ppb acting as sample species (where $\mathrm{C} 1$ here stands for the molecule having only one $\mathrm{C}$ atom, $\mathrm{C} 2$ for the fatty acid molecule having two $\mathrm{C}$ atoms, and so on) and lactic acid acting as buffer (at concentrations up to $30 \mathrm{ppb}$ ). Table 1 shows the

Table 1. Number of Carbons, Maximum Signal, Concentration of Vapors, and Overall Gain of the Different Fatty Acids Analyzed

\begin{tabular}{llll}
\hline Carbons & $\mathrm{S}_{\mathrm{s}}(\mathrm{cps})$ & $\mathrm{N}_{\mathrm{s}}(\mathrm{ppt})$ & Gain (cps/ppt) \\
\hline 1 & 70 & 1917.9 & $3.65 \mathrm{E}-02$ \\
2 & 129 & 1462.4 & $8.82 \mathrm{E}-02$ \\
3 & 1169 & 1181.7 & $9.89 \mathrm{E}-01$ \\
5 & 7840 & 853.94 & $9.18 \mathrm{E}+00$ \\
6 & 9042 & 749.93 & $1.21 \mathrm{E}+01$ \\
7 & 10838 & 656.21 & $1.65 \mathrm{E}+01$ \\
8 & 12409 & 591.94 & $2.10 \mathrm{E}+01$ \\
\hline
\end{tabular}

concentration of fatty acids and the gain (signal/concentration) produced when no lactic acid is introduced. Interestingly, bigger molecules are sensed more efficiently. This could be partly because bigger molecules, having lower mobility, have higher probability of ionization. As expected, Figure 4 shows how the signal of fatty acids falls as the concentration of lactic acid is increased.

In accordance with the results of Figure 3, theoretical curves are computed for $z_{b c}=1$. As for $\kappa$, in a first approximation, charge transfer will always occur if two particles collide and the charge affinity difference is favorable for the reaction. Under this hypothesis, the different reaction rate constants should be of the same order and $\kappa \sim 1$ (or $\kappa \sim 0$ if the reaction is not favorable). On the other hand, for favorable reactions, buffer vapors will have the highest charge affinity, followed by the sample vapors, while the charging agent has the lowest charge affinity. The charge to buffer reaction would therefore tend to be stronger than the sample to buffer reaction, and the ratio $\kappa$ would thus be smaller than one $(\kappa<1)$. Continuous and dashed theoretical lines in Figure 4 correspond, respectively, to $\kappa=0.1$ and $\kappa=0.3$. Post-processed experimental data are forced to meet theoretical curves at the point $(0,1)$, but there are no other restrictions. Most experimental data fall between the two theoretical curves $(\kappa=0.1$ and $\kappa=0.3)$, suggesting that both effects (1) charge transfer reactions from fatty acid to lactic acid, and (2) charging agent depletion is important and commensurable in our set-up.

\section{Conclusions}

The developed model hypothesizes that charging agents have constant composition. Although this holds true at least for highly conductive electrosprays producing very small droplets that quickly evaporate and produce ions, it is not necessarily always the case. Luckily, comparison of theoretical and experimental data suggest that in our specific 
configuration, ionization agents are highly mobile and that transfer reaction from sample ions (ionized by charge transfer reactions from charging agents) and charging agent depletion are commensurable effects for the case of a mixture of fatty acids and lactic acid. Although this is not conclusive evidence, our data support the hypothesis that SESI mechanism is, at least in this case, based on ion to molecule rather than droplet to molecule reactions.

Another interesting theoretical result is that low mobility charging agents would be much less affected by charge depletion. According to our model, when the mobility of the charging agents is much lower than the mobility of the ionized vapors, the behavior of the ionizer is perfectly linear until the charge is totally depleted. This interesting result suggests that SESI ionizers should use preferably low mobility charging agents to improve the linear response over a wider sample concentration range. An improved model including timevarying charging agent properties would be useful for a better understanding of this hypothetically more efficient regime.

\section{Acknowledgments}

The authors are most grateful to Juan F. de la Mora for his valuable insights and comments. They are also grateful to Pablo Martínez-Lozano for many fruitful conversations and to their colleagues at SEADM, and especially to Ernesto Criado, for his help during the experiments. This work was developed under the project EMOCION funded by the Plan AVANZA (Gobierno de España). The authors also thank Gonzalo F. de la Mora for his support for this project.

\section{Appendix I - Charging Vapors in the Linear Regime}

In a very first approximation, we shall consider only one reaction where the charging agent is composed of ions of only one type transferring their charge to the sample molecules to produce sample ions. As already noted by J. F. de la Mora [7], as the measured probability of ionization is very low, one can simplify the equations by assuming that the concentration of sample vapor remains constant and homogeneous (only a tiny part of the total molecules are ionized). Equations are also simplified by assuming that the concentration of charging ions is much higher than the concentration of sample ions. This implies that the electric field is only (or mainly) driven by the concentration of charging ions, and that the destruction rate of charging ions attributable to charge transfer reactions can also be ignored. These assumptions are reasonable in the limit of interest when one is trying to measure the sensitivity of a system because under such conditions the concentration of sample ions is very low. Applying these assumptions to the sample ion mass conservation equation of an infinitesimally small volume traveling at the velocity of the sample ions, and similarly to the charging ion mass conservation equation of a similarly and equally infinitesimal small volume traveling at the velocity of the charging ions yields:

$$
\begin{aligned}
& \frac{d n_{s}}{d \tau_{s}}=k_{c s} N_{s} n_{c}-n_{s} \nabla \cdot\left(Z_{s} \vec{E}+\vec{V}_{f}\right) \quad \quad(\mathrm{aI}-1 \mathrm{a}, \mathrm{b}) \\
& \frac{d n_{c}}{d \tau_{c}}=-n_{c} \nabla \cdot\left(Z_{c} \vec{E}+\vec{V}_{f}\right)
\end{aligned}
$$

Where $d \tau_{s}$ is the time required by the sample ions to travel an infinitesimally short distance $d l$ and $d \tau_{c}$ is the time required by the charging ions to travel the same similarly infinitesimal short distance $d l$. Note here that $d n / d \tau$ in equations (aI-1a, b), not to be confused with Eulerian notation, refers to the Lagrangian time derivative of the concentration of an infinitesimally small volume following the ions at the same speed of the ions. Although $d n_{s} / d \tau$ differs from zero, the configuration might be in a stationary state. Because each type of ion has a different mobility, the streamlines of species are different and $\tau$ cannot be directly simplified. But under special circumstances where $d \tau_{s}$ and $d \tau_{c}$ are related, equations (aI-1a, b) can be further simplified. If the fluid velocity is negligible or if both the sample ion and the charging ion have the same mobility, the charging and the sample ions follow the same trajectory even though they might travel at different speeds. Under such circumstances, $d \tau_{s}$ and $d \tau_{c}$ are related as follows:

$$
\begin{aligned}
& d \tau_{c}=d \tau \frac{Z_{s}}{Z_{c}} \\
& d \tau_{s}=d \tau
\end{aligned}
$$

and the variable $\tau$ can be further simplified by dividing equations (aI-1a) and (aI-1b), using the relations $\nabla \vec{E}=n_{c} e / \varepsilon_{0}$, and (aI-2), and assuming that the flow is incompressible:

$$
\frac{d n_{s}}{d n_{c}}=\frac{k_{c s} N_{s} n_{c}-n_{s} Z_{s} \frac{e}{\varepsilon_{0}} n_{c}}{\frac{Z_{s}}{Z_{c}}\left(-n_{c} Z_{c} \frac{e}{\varepsilon_{0}} n_{c}\right)}
$$

Rearranging the expression of equation (aI-3) and using the parameter $n_{s}^{*}=N_{s} \frac{k_{c s} \varepsilon_{0}}{Z_{s^{*}} \cdot e}$ (having dimensions of concentration), yields a very simple equation:

$$
\frac{d n_{s}}{n_{s}-n_{s}^{*}}=\frac{d n_{c}}{n_{c}}
$$

that can be easily integrated for a given initial condition $\left(n_{s 0}\right.$ and $n_{c 0}$ ), leading to the result already presented in the main text as equation 4 :

$$
n_{s}=n_{s}^{*}\left(1-\frac{n_{c}}{n_{c 0}}+n_{s 0} \frac{n_{c}}{n_{c 0}}\right)
$$




\section{Appendix II - Charging Vapors Comprising a Mayor Species and a Minor Species, Theoretical Approach}

In the regime of interest, the electric field is produced by both the charging and the buffer ions, and the production destruction of charging ions. The amount of sample vapors will be considered sufficiently small to assume that its effect on both the neutralization of charging ions and the electric field is negligible. Sample ions are produced by transfer reaction from charging ion to sample vapor and are neutralized by charge reactions from sample ions to neutral buffer vapors. The set of equations governing the behavior of the charging, the buffer, and the sample ions become:

$$
\begin{aligned}
& \frac{d n_{s}}{d \tau_{s}}=k_{c s} N_{s} n_{c}-k_{s b} n_{s} N_{b}-n_{s} \nabla \cdot\left(Z_{s} \vec{E}+\vec{V}_{f}\right) \\
& \frac{d n_{c}}{d \tau_{c}}=-k_{c b} N_{b} n_{c}-n_{c} \nabla \cdot\left(Z_{c} \vec{E}+\vec{V}_{f}\right) \\
& \frac{d n_{b}}{d \tau_{b}}=k_{c b} N_{b} n_{c}-n_{b} \nabla \cdot\left(Z_{b} \vec{E}+\vec{V}_{f}\right)
\end{aligned}
$$

$$
\nabla \cdot \vec{E}=\frac{e}{\varepsilon_{0}}\left(n_{c}+n_{b}\right)
$$

where $n_{b}$ is the concentration of buffer ions, $N_{b}$ is the concentration of buffer vapors, $Z_{b}$ is the mobility of buffer ions, $k_{c b}$ and $k_{s b}$ are the reaction rate constants for charge transfer from the charging ions to the buffer molecule, and from the sample ions to the buffer molecules, respectively, and $d \tau_{b}$ is the time required by the buffer ions to travel an infinitesimally short distance $d l$.

Again, if the fluid velocity is negligible, or if all the sample, buffer, and charging ions have the same mobility, all ions follow the same trajectory even though they might travel at different speeds. Under such circumstances, $d \tau_{s}, d \tau_{b}$, and $d \tau_{c}$ are related to $d \tau$ similarly as in equation (aI-2):

$$
\begin{aligned}
d \tau_{c} & =d \tau \frac{Z_{b}}{Z_{c}} \\
d \tau_{s} & =d \tau \frac{Z_{b}}{Z_{s}} \\
d \tau_{b} & =d \tau
\end{aligned}
$$$$
(\mathrm{aII}-3 \mathrm{a}, \mathrm{b}, \mathrm{c})
$$
of buffer ions is also reflected in a non-negligible

assuming that the velocity field is incompressible:

$$
\begin{aligned}
\frac{d n_{b}}{d n_{c}} & =\frac{n_{c} n_{b}^{*}-n_{b}\left(n_{b}+n_{c}\right)}{-n_{c} n_{b}^{*} \frac{Z_{b}}{Z_{c}}-n_{c}\left(n_{b}+n_{c}\right)} \\
\frac{d n_{s}}{d n_{c}} & =\frac{n_{s}^{*} n_{c}-n_{b}^{\prime} n_{s}-n_{s}\left(n_{b}+n_{c}\right)}{-n_{c} n_{b}^{*} \frac{Z_{b}}{Z_{c}}-n_{c}\left(n_{b}+n_{c}\right)}
\end{aligned}
$$

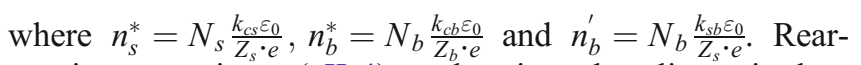
ranging equations (aII-4) and using the dimensionless parameters $\eta_{s}=n_{s} / n_{s}^{*}, \eta_{c}=n_{c} / n_{b}^{*}, \eta_{b}=n_{b} / n_{b}^{*} Z b c=Z_{b} /$ $Z_{c}$, and $\kappa=k_{s b} / k_{c b}$ yields:

$$
\begin{aligned}
\frac{d \eta_{b}}{d \eta_{c}} & =\frac{\eta_{c}-\eta_{b}\left(\eta_{c}+\eta_{b}\right)}{-\eta_{c} z_{b c}-\eta_{c}\left(\eta_{c}+\eta_{b}\right)} \\
\frac{d \eta_{s}}{d \eta_{c}} & =\frac{\eta_{c}-\eta_{s} z_{b c} \kappa-\eta_{s}\left(\eta_{c}+\eta_{b}\right)}{-\eta_{c} z_{b c}-\eta_{c}\left(\eta_{c}+\eta_{b}\right)}
\end{aligned}
$$

This set of equations can be numerically integrated, given a set of initial conditions $\left(n_{s 0}, n_{b 0}\right.$, and $\left.n_{c 0}\right)$. For the case of an electrospray having a singular source of charging ions, one would expect to find the asymptotic behavior already predicted by de la Mora [7] near the cone apex where concentration of charging ions tends toward infinity. To recover this behavior, equations (aII-5) are integrated numerically by starting the integration at the source $\eta_{s} \rightarrow \infty$ with the equilibrium initial condition that $\eta_{s} \rightarrow 1$ and $\eta_{b} \rightarrow 1$.

Solving these equations for $\kappa=0$ and for different values of $z_{b c}$ show that the dependence of the concentration of buffer ions as charging ion concentration varies is lower for $z_{b c}>>1$. This result suggests that a SESI ionizer using low mobility charging agents (such as droplets rather than single ions) would improve the linear range of the ionizer.

In the limit when $z_{b c} \kappa \rightarrow \infty$, the concentration of sample ions falls when the fraction of buffer ions is still very low. This means that the main mechanism reducing the sample ion concentration is charge transfer reaction from the sample ions to the buffer molecules rather than charging agent depletion. Obviously, equations (aII-5) cannot be integrated in this regime, but a new set of equations can be rewritten for this more special case where the only effect of the buffer vapor concentration is to stead charge from the sample:

$$
\begin{aligned}
\frac{d n_{s}}{d \tau_{s}} & =k_{c s} N_{s} n_{c}-k_{s b} n_{s} N_{b}-n_{s} \nabla \cdot\left(Z_{s} \vec{E}+\vec{V}_{f}\right) \\
\frac{d n_{c}}{d \tau_{c}} & =n_{s} \nabla \cdot\left(Z_{c} \vec{E}+\vec{V}_{f}\right)
\end{aligned}
$$

and the variable $\tau$ can be further simplified by dividing equations (aII-1c) and (aII-1b), and equations (aII-1a) and (aII-1b), using the relations (aII-2) and (aII-3), and

$$
\nabla \cdot \vec{E}=\frac{e}{\varepsilon_{0}} n_{c}
$$


Proceeding as before to eliminate the variables $d \tau_{s}$ and $d \tau_{c}$ yields:

$$
\frac{d n_{s}}{d n_{c}}=\frac{n_{s}^{*} n_{c}-n_{b}^{\prime} n_{s}-n_{s} n_{c}}{n_{c}^{2}}
$$

and this equation can be further simplified using the dimensionless parameters $\eta_{s}=n_{s} / n_{s}^{*}$ and $\mu_{c}=n_{c} / n_{b}^{\prime}$ (where $n_{b}^{\prime}=N_{b} \frac{k_{s b} \varepsilon_{0}}{Z_{s} \cdot e}$. Note that $\mu_{c}=\eta_{c} z_{b c} \kappa$ ).

$$
\frac{d \eta_{s}}{d \mu_{c}}=\frac{\mu_{c}-\eta_{s}-\eta_{s} \mu_{c}}{\mu_{c}^{2}} \quad(\mathrm{aII}-8)
$$

Equation (aII-8) can be integrated analytically. Imposing the condition that the solution is bound when $\mu_{c} \rightarrow \infty$, the solution for equation (aII-8) is equation (aII-9), which also recovers the result already expected when the concentration of charging ions is much higher than the concentration of buffer ions.

$$
\eta_{s}=\mu_{c}\left(1-e^{\frac{1}{\mu_{c}}}\right)
$$

For a given ionization configuration, the concentration of charging ions is constant (note that in the proposed limit, charging agent depletion is negligible) $n_{c}=n_{t o t}$, and the ratio $\mu_{c}$ is inversely proportional to the buffer vapor concentration. In the limit when $\mu_{c} \rightarrow \infty$ (very low concentration of buffer vapors), $\eta_{s}$ tends to one, and in the limit when $\mu_{c} \rightarrow 0$ (very high concentration of buffer vapors), $\eta_{s}$ tends toward zero.

\section{Appendix III - Charging Vapors when the Electrospray Plume Produces Charging Agents, but Also Charged Particles which Reduce Performance}

For simplicity, we shall consider only one sample species at very low concentration. Equations are also simplified assuming that the concentration of electrosprayed ions is much higher than the concentration of sample ions and, therefore, that the electric field is only driven by the concentration of electrosprayed ions, and that the destruction rate of charging ions is also negligible. These simplifications yield the following set of equations:

$$
\begin{aligned}
& \frac{d n_{s}}{d \tau_{s}}=k_{c s} N_{s} n_{c}-n s \nabla \cdot\left(Z_{s} \vec{E}+\vec{V}_{f}\right) \\
& \frac{d n_{c}}{d \tau_{c}}=-n_{c} \nabla \cdot\left(Z_{c} \vec{E}+\vec{V}_{f}\right) \\
& \frac{d n_{i}}{d \tau_{i}}=-n_{i} \nabla \cdot\left(Z_{i} \vec{E}+\vec{V}_{f}\right) \\
& \nabla \cdot \vec{E}=\frac{e}{\varepsilon_{0}}\left(n_{c}+\sum n_{i}\right)
\end{aligned}
$$$$
(\mathrm{aIII}-1 \mathrm{a}, \mathrm{b}, \mathrm{c})
$$$$
(\text { aIII - 2) }
$$$$
\begin{aligned}
& E \\
& V_{f} \\
& \tau
\end{aligned}
$$

where the useless electrosprayed ions are denoted with subscript ${ }_{i}, n_{i}$ is the concentration of the charged particle of type $i, Z_{i}$ is the mobility of the charged particle $i$ and $d \tau_{i}$ is the time required by the $i$ ions to travel an infinitesimally short distance $d l$. If the fluid velocity is negligible, or if all the sample, buffer, and charging ions have the same mobility, all ions follow the same trajectory even though they might travel at different speeds. Under such circumstances, $d \tau_{s}, d \tau_{i}$, and $d \tau_{c}$ are related to $\tau$, similarly as in equations (aI-2):

$$
\begin{aligned}
d \tau_{c} & =d \tau \frac{Z_{s}}{Z_{c}} \\
d \tau_{i} & =d \tau \frac{Z_{s}}{Z_{i}} \\
d \tau_{s} & =d \tau
\end{aligned}
$$

and the variable $\tau$ can be further simplified dividing equations (aIII-1c) and (aIII-1c), and equations (aIII-1c) and (aIII-1c), using the relations (aIII-2) and (aIII-3), and assuming that the velocity field is incompressible:

$$
\begin{aligned}
\frac{d n_{s}}{d n_{c}} & =\frac{n_{s}^{*} n_{c}-n_{s}\left(n_{c}+\sum n_{i}\right)}{-n_{c}\left(n_{c}+\sum n_{i}\right)} \\
\frac{d n_{i}}{d n_{c}} & =\frac{n_{i}}{n_{c}}
\end{aligned}
$$

Equations (aIII-4b) can be directly integrated yielding $n_{i} /$ $n_{c}=C_{i}$, where $C_{i}$ is the concentration ratio of each species that will remain constant as long as the composition of the plume is in equilibrium; equation (aIII-4a) can also be integrated analytically introducing the values $C_{i}$, giving the result already presented in the main text as equation 11 :

$$
n_{s}=\frac{n_{s}^{*}}{1+\sum C_{i}}\left(1-\frac{n_{c}}{n_{c 0}}\right)+n_{s 0} \frac{n_{c}}{n_{c 0}} \quad(\text { aIII }-5)
$$

List of Symbols

\begin{tabular}{ll}
\hline Symbol & Definition \\
$p_{i}$ & Ionization probability \\
$n_{s}$ & Concentration of sample ions \\
$N_{s}$ & Concentration of sample neutral vapors \\
$e$ & The charge of the ion \\
$\varepsilon_{0}$ & Permittivity of the vacuum \\
$Z_{s}$ & Mobility of the sample ion \\
$k_{c s}$ & Reaction rate coefficient for the charge transfer \\
& reaction from the charging ions to the sample \\
$E$ & molecule \\
$V_{f}$ & Electric field \\
$\tau$ & Fluid velocity of the gas dragging the ions \\
& Time for each infinitesimally small volume \\
& following each type of ionic streamline at a \\
$n_{s}^{*}=N_{s} \frac{k_{c s} \varepsilon_{0}}{Z_{s} \cdot e}$ & velocity $V_{f}+Z E$ \\
$n_{s}$ &
\end{tabular}




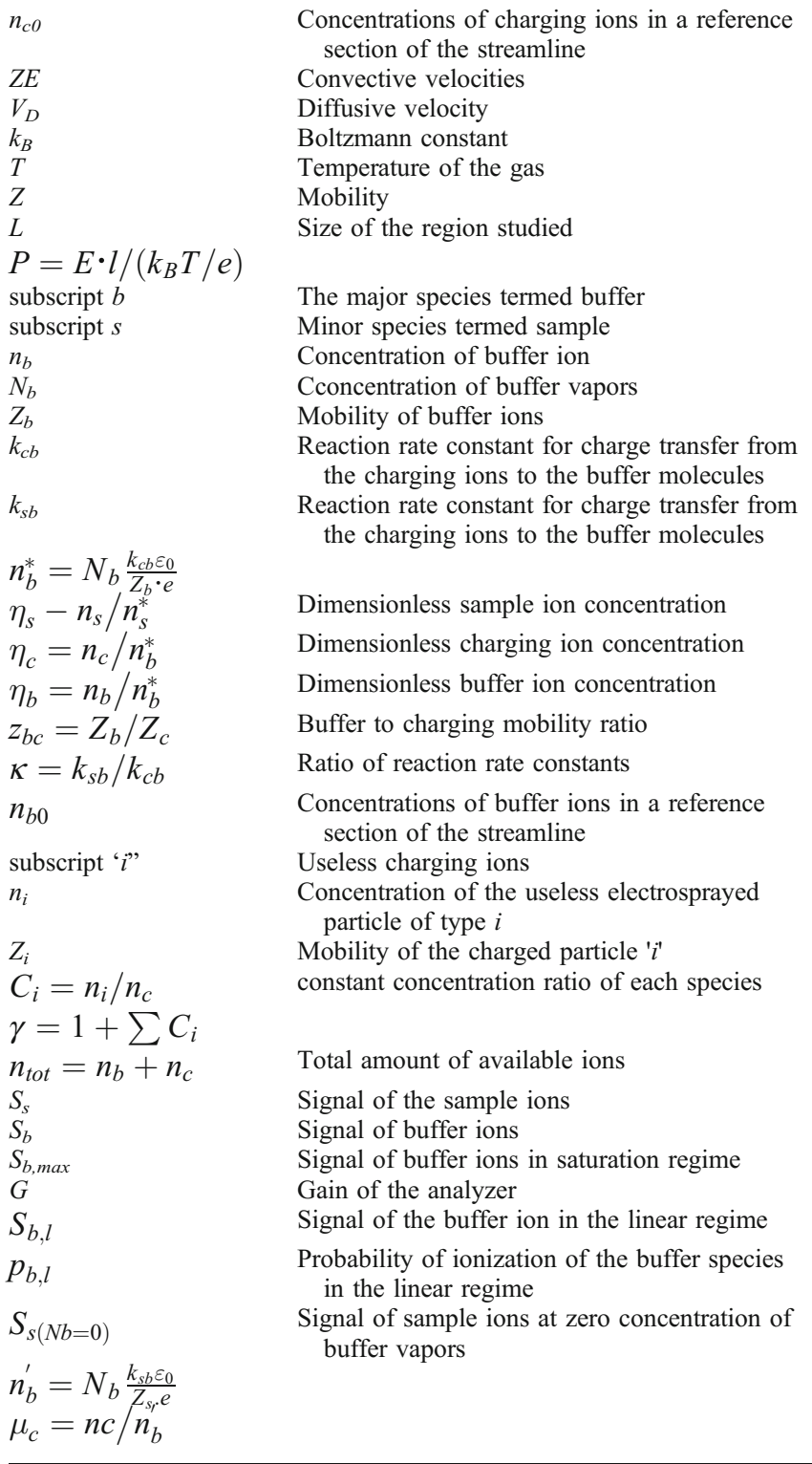

\section{References}

1. Whitehouse, C.M.; Levin, F.; Meng, C.K.; Fenn, J.B. Proceedings of the 34th ASMS Conference on Mass Spectrometry and Allied Topics, Denver, CO, 1986; p. 507.

2. Fuerstenau, S.; Kiselev, P.; Fenn, J. B. ESIMS in the analysis of trace species in gases. Proceedings of the 47th ASMS Conference on Mass Spectrometry, Dallas TX, 1999.

3. Fuerstenau, S. Aggregation and fragmentation in an electrospray ion source. Ph.D. Thesis, Department of Mechanical Engineering, Yale University, 1994.

4. Wu, C., Siems, W.F., Hill Jr., H.H.: Secondary electrospray ionization ion mobility spectrometry/mass spectrometry of illicit drugs. Anal. Chem. 72, 396-403 (2000)

5. Martínez-Lozano, P.; Rus, J.; Fernández de la Mora, G.; Hernández, M.; Fernández de la Mora J. Detection of explosive vapors below part per trillion concentrations with electrospray charging and atmospheric pressure ionization mass spectrometry (API-MS). J. Am. Soc. Mass Spectrom. 20, 287-294

6. Mesonero, E.; Sillero, J.; Hernandez, M.; Fernandez de la Mora, J. Secondary electrospray ionization detection of explosive vapors below $0.02 \mathrm{ppt}$ on a triple quadrupole with an atmospheric pressure source. Proceedings of the 57th ASMS Conference, Philadelphia, PA(USA), May 2009.

7. Fernandez de la Mora, J. Ionization of napor molecules by an electrospray cloud; special issue of the International Journal of Mass Spectrometry (Samy El-Shall and David Muddiman, Editors) to honor the Science and Impact of John B. Fenn; May/5/2010.

8. Martínez-Lozano P.; Criado E.; Vidal G. Mechanistic study on the ionization of trace gases by an electrospray plume. Int. J. Mass Spectrom. 313, 21-29 (2011)

9. Martínez-Lozano, P., Fernández de la Mora, J.: On-line detection of fatty acid vapors released by human skin. J. Am. Soc. Mass Spectrom 20 (6), 1060-1063 (2009)

10. Martínez-Lozano, P.: Mass spectrometric study of cutaneous volatiles by secondary electrospray ionization. Int. J. Mass Spectrom. 282(3), 128-132 (2009)

11. U.S. Patent application 11/732,770; Martínez-Lozano P., Fernandez de la Mora J. Method for detecting volatile species of high molecular weight. April 4, 2006.

12. Hogan C. J. Jr.; Fernández de la Mora, J. Tandem ion mobilitymass spectrometry (IMS-MS) study of ion evaporation from ionic liquid-cetonitrile nanodrops. Phys. Chem., Chem. Phys. (36), 8079-8090 (2009)

13. Fernández de la Mora, J.: The effect of charge emissions from electrified liquid cones. J Fluid Mech 243, 561-574 (1992) 